\title{
Is the Electron Radiation Length Constant at High Energies?
}

\author{
H. D. Hansen, ${ }^{1}$ U. I. Uggerh $\varnothing j,{ }^{1}$ C. Biino, ${ }^{2}$ S. Ballestrero, ${ }^{3}$ A. Mangiarotti, ${ }^{3}$ P. Sona, ${ }^{3}$ T. J. Ketel, ${ }^{4}$ and Z. Z. Vilakazi ${ }^{5}$ \\ ${ }^{1}$ Department of Physics and Astronomy, University of Aarhus, Denmark \\ ${ }^{2}$ CERN, Geneva, Switzerland \\ ${ }^{3}$ University of Florence, Florence, Italy \\ ${ }^{4}$ Free University, Amsterdam, The Netherlands \\ ${ }^{5}$ University of Cape Town, Cape Town, South Africa
}

(Received 7 April 2003; published 1 July 2003)

\begin{abstract}
Experimental results for the radiative energy loss of 149, 207, and $287 \mathrm{GeV}$ electrons in a thin $\mathrm{Ir}$ target are presented. From the data we conclude that at high energies the radiation length increases in accordance with the Landau-Pomeranchuk-Migdal (LPM) theory and thus electrons become more penetrating the higher the energy. The increase of the radiation length as a result of the LPM effect has a significant impact on the behavior of high-energy electromagnetic showers.
\end{abstract}

DOI: 10.1103/PhysRevLett.91.014801

In the early 1950s, Ter-Mikaelian postulated the existence of the so-called formation length [1] for the emission of radiation. This is, loosely speaking, the length it takes to separate the photon from the electron by one wavelength such that the photon can be considered "formed." If for some reason the electron is influenced during this formation, the yield of photons may increase as when electrons traverse crystals [2] or it may be reduced due to destructive interference. The formation length is the basic parameter of the LandauPomeranchuk-Migdal (LPM) effect [3,4], which predicts a reduction of photon yield due to multiple scattering in the formation zone.

A beautiful series of detailed experiments was performed at Stanford Linear Accelerator Center (SLAC) to examine the LPM effect by use of electrons of 8 and $25 \mathrm{GeV}$ [5]. However, only the lower $500 \mathrm{MeV}$ of the photon spectrum was recorded, and, thus, no conclusion could be drawn with respect to a possible variation of the radiation length $X_{0}$ based on the experimental data.

The present investigation gives experimental evidence for the energy dependence of $X_{0}$. The yield of bremsstrahlung photons is compared to theoretical calculations over essentially the complete energy range.

The LPM effect - besides being interesting in itself as a basic QED process - is relevant in several connections. In the first place, it may have a significant impact on the behavior of air showers in the neighborhood of the Greisen-Zatsepin-Kuz'min cutoff of high-energy photons [6,7]. Second, the LPM effect in QED processes may have a parallel in suppression of gluons in QCD processes [8,9]. Finally, an electromagnetic shower initiated by an electron may develop over a characteristic length that is increased substantially compared to the nominal $X_{0}$ as well as having a different composition. As an example, in lead-tungstate crystals, the shower develops corresponding to a radiation length that is longer than the nominal $X_{0}$ by as much as $2.5 \%, 10 \%$, or $26 \%$ for energies $0.2,1$, or $4 \mathrm{TeV}$, respectively.
Since the SLAC experiments, the theory of the LPM effect has evolved substantially: several groups have calculated LPM suppression using different approaches, among these Baier and Katkov [10], Blankenbecler and Drell [11], Zakharov [12] and Shul'ga and Fomin [13]. Furthermore, a comprehensive review by Klein has appeared [14].

The formation length is given for emission of radiation as [15]:

$$
l_{\mathrm{f}}=\frac{2 \gamma^{2} c}{\omega^{*}} \quad \text { with } \omega^{*}=E \frac{\omega}{E-\hbar \omega} \simeq \omega
$$

where $E=\gamma m c^{2}$ and $\hbar \omega$ are the energy of the electron and the photon, respectively, $m$ is the rest mass of the electron, $\gamma$ is the Lorentz factor, and $c$ is the speed of light [15]. Note that the last approximation in Eq. (1) is in the classical or recoilless limit, $\hbar \omega \ll E$.

The length over which a particle statistically scatters an angle $1 / \gamma$ in an amorphous material due to multiple Coulomb scattering is given by

$$
l_{\gamma}=\frac{\alpha}{4 \pi} X_{0},
$$

where $\alpha$ is the fine-structure constant. Since the majority of radiation emission takes place within a cone of opening angle $1 / \gamma$ to the direction of the electron, destructive interference may result if the electron scatters outside this zone. So, if half the formation length exceeds the length $l_{\gamma}$ (in the convention of Ref. [13]), the emission probability decreases. Equation (1) combined with Eq. (2) leads to the onset of the LPM effect at energies:

$$
\hbar \omega_{\mathrm{LPM}}^{\mathrm{q}}=\frac{E^{2}}{E+E_{\mathrm{LPM}}} \quad\left(\hbar \omega_{\mathrm{LPM}}^{\mathrm{c}} \simeq \frac{E^{2}}{E_{\mathrm{LPM}}}\right),
$$

where $E_{\mathrm{LPM}}=m c^{2} X_{0} / 4 \pi a_{0}=7.68 X_{0} \mathrm{TeV} / \mathrm{cm}$ and $a_{0}$ is the Bohr radius. The value in parentheses denotes the classical (recoilless) limit. 
We note that $\hbar \omega_{\text {LPM }}$ is roughly proportional to $E^{2}$ at moderate energies, which is why electrons of, e.g., $207 \mathrm{GeV}$ affect the photon spectrum much more than at the SLAC energies of 8 or $25 \mathrm{GeV}$. Moreover, for the latter the LPM effect is described with sufficient accuracy for all targets by use of $\hbar \omega_{\mathrm{LPM}}^{\mathrm{c}}$ since, e.g., $\hbar \omega_{\mathrm{LPM}}^{\mathrm{q}} / \hbar \omega_{\mathrm{LPM}}^{\mathrm{c}}=$ 1.01 for $25 \mathrm{GeV}$ electrons in gold. This is barely detectable and justifiably neglected by the SLAC group, who quote a $3.5 \%$ total systematic error.

The LPM cross section for bremsstrahlung is given by Migdal as [3,14]:

$$
\begin{aligned}
\frac{d \sigma_{\mathrm{LPM}}}{d \hbar \omega}= & \frac{4 \alpha r_{e}^{2} \xi(s)}{3 \hbar \omega}\left\{y^{2} G(s)+2\left[1+(1-y)^{2}\right] \phi(s)\right\} Z^{2} \\
& \times \ln \left(184 Z^{-1 / 3}\right),
\end{aligned}
$$

where $G(s), \phi(s)$, and $\xi(s)$ are functions of $s=$ $\sqrt{E_{\mathrm{LPM}} \hbar \omega / 8 E(E-\hbar \omega) \xi(s)}$, i.e., $\xi(s)$ is given recursively but can be well approximated; see, e.g., Refs. [13,15]. Here $y$ denotes the fractional photon energy $\hbar \omega / E, Z$ the nuclear charge of the target, and $r_{e}=\alpha^{2} a_{0}$ the classical electron radius. In the limit $G(s)=\phi(s)=1$ the Bethe-Heitler cross section is obtained. The resulting suppression of low energy photons has an analogue in the case of pair production where symmetric pairs are suppressed due to the formation length being proportional to $\xi_{+} \xi_{-}$with $\xi_{ \pm}=E_{e^{ \pm}} / \hbar \omega$. For a thorough treatment of the subject, see [13].

A possible compensation to the LPM effect which would appear at high energies has been discussed by Bell [17]. This "compensation" would leave the radiation length constant. It is thus not a priori a sign of changing $X_{0}$ if the lower few percent of the radiation spectrum is suppressed. However, Bell's calculations are based on classical theory and he even expressed his own doubts: "However, the compensating enhancement is in a region where classical theory is quite wrong, so that as an essentially quantum effect the increase of penetrating power $[\ldots]$ may indeed exist."

Other suppression effects have shown to be present at lower photon energies (transition radiation, dielectric suppression - the Ter-Mikaelian effect) [5], but the characteristic photon energy below which these effects appear is very low, around $50 \mathrm{MeV}$ for $300 \mathrm{GeV}$ electrons, and they have essentially no observable influence on the results presented here. One other effect approaches the region of interest in the present experiment, however. Since the formation length increases with decreasing photon frequency, at a certain point the formation zone extends beyond the thickness of the foil. In this case, the radiation yield also becomes suppressed; see, e.g., [17]. Setting the thickness $\Delta x=l_{\mathrm{f}}$, the effect appears for photon energies below $\hbar \omega=E /\left(1+\Delta x / 2 \gamma \lambda_{c}\right)$, where $\lambda_{c}=\alpha a_{0}$. As an example, electrons of $E=287 \mathrm{GeV}$ yield a suppression for photon energies below $\hbar \omega=$ $0.9 \mathrm{GeV}$ in $\mathrm{Ir}$ and $0.2 \mathrm{GeV}$ in $\mathrm{Cu}$, i.e., just below the observed photon energies in this experiment.
The experiment was performed in the $\mathrm{H} 2$ beam line of the CERN Super Proton Synchrotron in a tertiary beam of electrons with variable energy in the range $10-300 \mathrm{GeV}$, but with low intensity for very high and very low energies. The fraction of particles heavier than electrons in the beam is very low, estimated to $\sim 10^{-3}$. A schematic drawing of the setup is shown in Fig. 1. The incident electron beam is defined by three scintillator counters, $\mathrm{S} 1, \mathrm{~S} 2$, and $\overline{\mathrm{S} 3}$, and the position and direction of each electron is found from its impact on drift chambers DC1 and DC2 before the dipole magnet, $\mathrm{B} 8$, and in DC3 and/ or DC4 after this magnet. In front of $\overline{\mathrm{S} 3}$ the target of about $4 \% X_{0}$ is placed. The magnet and the drift chambers enable energy tagging of the photons emitted in the target. The photons are finally intercepted in a lead glass detector (LG). Each DC has a resolution of $\sigma \simeq 100 \mu \mathrm{m}$ and the distances are such that the resulting angular resolution is $\sim 10 \mu \mathrm{rad}$. For the tagging, DC3 is used for maximum acceptance while DC4 provides the optimum resolution for low energy photons. The relative resolution of the $\mathrm{LG}$ is $\sigma / E \simeq 0.16 / \sqrt{E[\mathrm{GeV}]}+$ $0.0029+1.2 \times 10^{-4} E[\mathrm{GeV}]$, which is found by directing electron beams of various energies in the range 10$287 \mathrm{GeV}$ into the LG. For the highest energies, nonlinearity in the response of the LG becomes important and is taken into account in the analysis. The low energy cutoff of the lead glass spectrum was set to $2 \mathrm{GeV}$ to avoid influence from the pedestal. Consistency of the LG calibration and the tagging system was confirmed by switching B8 on and off. The resolution of the DC4 tagging system is proportional to $(E-\hbar \omega)(E / \hbar \omega-1)$ and is about $40 \%$ at $2 \mathrm{GeV}$. Thus, we consider photons with energies above $2 \mathrm{GeV}$ only.

The contributions measured with an empty target at each energy, corresponding to about $1 \% X_{0}$, have been subtracted from the data. To determine the total efficiency of the LG calorimeter as a function of total radiated energy, a carbon target was used. As discussed below, the LPM effect can be considered absent in the detected range for carbon. The experimental result was compared to a simulation based on the standard Bethe-Heitler expression to extract the efficiency. Simulations were performed by the use of a modified [19] version of GEANT [20] which takes into account the LPM effect according to Eq. (4). The standard Bethe-Heitler spectrum was obtained by setting $E_{\mathrm{LPM}}=10^{9} \mathrm{GeV}$. The correction due to the efficiency was found to be small for all photon energies, i.e., less than $10 \%$ and typically $4 \%-5 \%$. An efficiency different from one can be due to a number of geometrical factors, for instance, pair production in the He bag where the opening angle of the pair is such that it misses the LG or an excess of counts due to interaction of the spent electron with the He-bag vessel that may be partly intercepted by the LG. In order to reduce the influence of pileup of several sequentially emitted photons, a thin Ir target thickness of $0.128 \mathrm{~mm}$ (about 


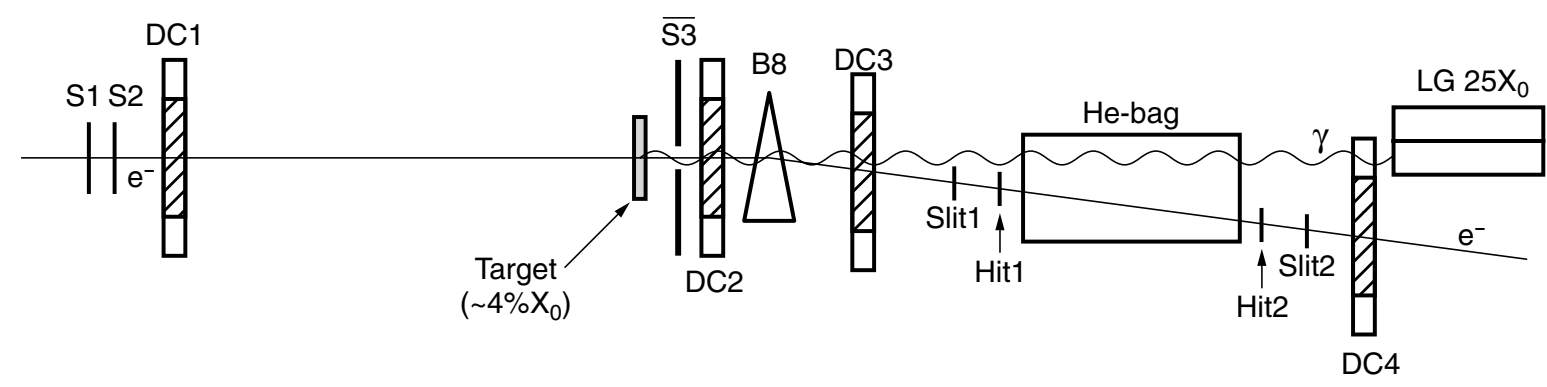

FIG. 1. A schematic drawing of the setup used in the CERN LPM experiment. The total length of the setup is about $80 \mathrm{~m}$.

$\left.0.04 X_{0}\right)$ was chosen. This means that the average photon yield above the threshold of $2 \mathrm{GeV}$ is about $10 \%$; thus, we expect a very low probability for two-photon emission. The energies of the onset of the LPM effect are calculated with Eq. (3) for the quantum case $(q)$ and for the classical approximation $(c)$. For $287 \mathrm{GeV}$ electrons in $\operatorname{Ir} \hbar \omega_{\mathrm{LPM}}^{\mathrm{q}}=$ $32 \mathrm{GeV}$ and $\hbar \omega_{\mathrm{LPM}}^{\mathrm{c}}=37 \mathrm{GeV}$, while in $\mathrm{C} \hbar \omega_{\mathrm{LPM}}^{\mathrm{q}}=$ $0.44 \mathrm{GeV}$ and $\hbar \omega_{\mathrm{LPM}}^{\mathrm{c}}=0.44 \mathrm{GeV}$, respectively. Thus, as the experiment is sensitive only to photon energy sums above $2 \mathrm{GeV}$, it is fair to consider the carbon target as a target without LPM effect in the detected range. The value of the nominal $X_{0}$ of each of the targets was determined by weighing and measuring the foils to obtain the areal density and using Tsai's tabulated values for the unit radiation length [21]. The resulting values are $\Delta t_{\mathrm{C}} / X_{0}=4.14 \pm 0.05 \%$ and $\Delta t_{\mathrm{Ir}} / X_{0}=4.36 \pm 0.10 \%$ for $\mathrm{C}$ and Ir, respectively. The density of the carbon target was $1.74 \mathrm{~g} / \mathrm{cm}^{3}$.

In Figs. 2(a)-2(c) are shown the total radiated energy spectra (logarithmic binning with 25 bins/decade) obtained for 287, 207, and $149 \mathrm{GeV}$ electrons in the Ir target. The dotted lines denote the values obtained by a GEANT simulation of the spectrum of the total radiated energy based on a Bethe-Heitler bremsstrahlung spectrum for the nominal thickness in units of $X_{0}$, i.e., excluding any suppression effects. The solid lines result from the simulations performed with the modified version of GEANT, which takes into account the LPM effect. All simulations were performed using a lower energy cutoff of $0.1 \mathrm{GeV}$ to include the effect of pileup and no scaling of target thickness has been applied. The LPM effect suppression in Ir appears clearly at low photon energies in very good agreement with the quantum mechanical prediction. Furthermore, the agreement is good over the entire spectrum for all three energies, although there is a slight tendency for the data to lie below the simulation for the measurement with $149 \mathrm{GeV}$ electrons. The suppression amounts to as much as a factor of 2 for the lowest photon energies investigated and the onset of the LPM effect is found at photon energies approximately proportional to $E^{2}$ as expected from Eq. (3).

We note that the effective radiation length is proportional to the inverse of the areas of the spectra in Figs. 2(a)-2(c). Therefore, if the radiation length would be constant, the clear "lack" of radiation observed at low energies should be compensated at higher energies. To show this more clearly, we present in Fig. 3 the power spectrum for $287 \mathrm{GeV}$, i.e., the number of events per unit energy multiplied by the total radiated energy and with linear binning. The integral of Fig. 3 divided by $287 \mathrm{GeV}$
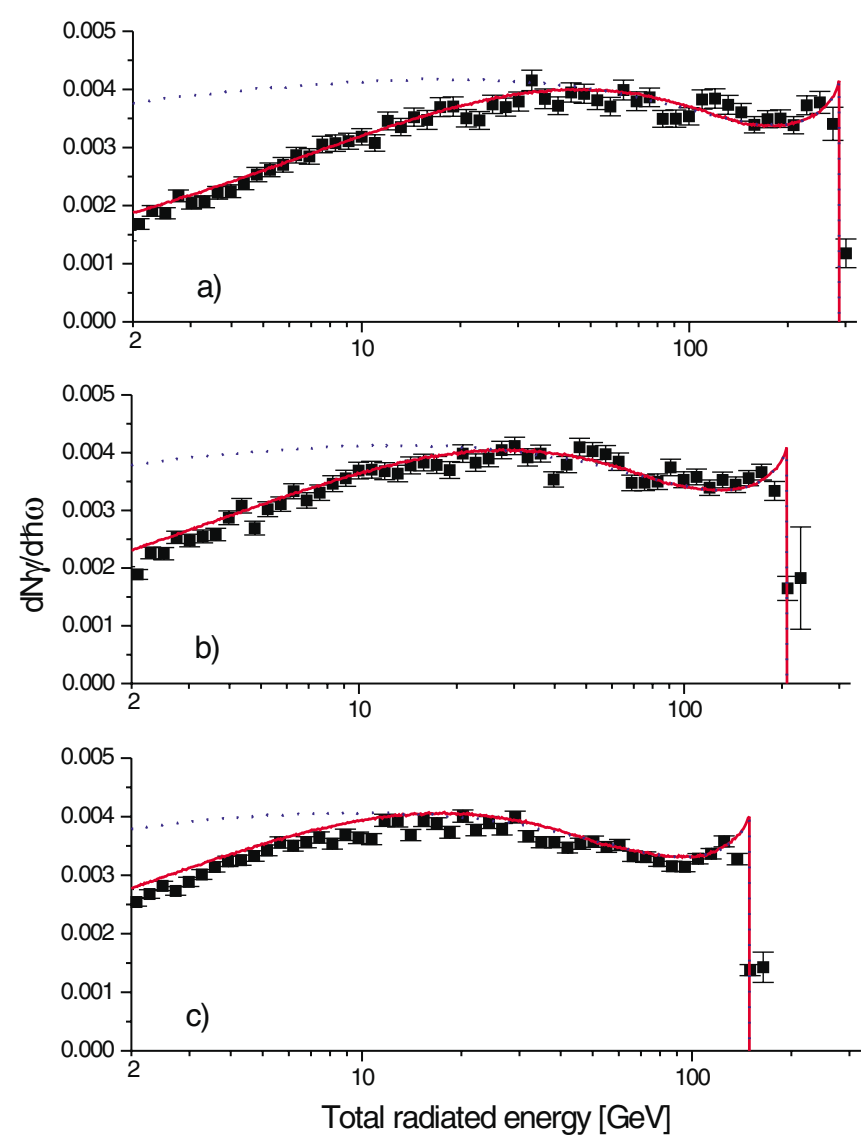

FIG. 2 (color online). Bremsstrahlung spectrum, $d N_{\gamma} / d \hbar \omega$, for (a) 287, (b) 207, and (c) $149 \mathrm{GeV}$ electrons on $0.128 \mathrm{~mm} \mathrm{Ir}$ $\left(4.36 \% X_{0}\right)$. The total radiated energy, $\hbar \omega$, is presented in logarithmic bins (25 per decade) and plotted on a logarithmic scale. The vertical scale is normalized to the number of incoming electrons. The dotted line is the result of a simulation based on a pure Bethe-Heitler spectrum while the solid line includes the LPM suppression. 


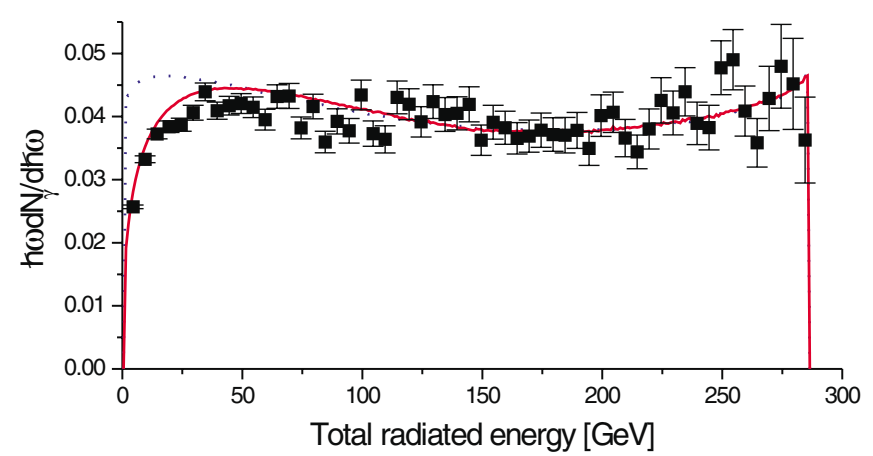

FIG. 3 (color online). The power spectrum, $\hbar \omega d N_{\gamma} / d \hbar \omega$, of the data from Fig. 2(a), but with linear bins. The dotted line is the result of a simulation based on a pure Bethe-Heitler spectrum while the solid line includes the LPM suppression.

is equal to the thickness in units of the effective radiation length.

By integration of each of the two intervals split by $\hbar \omega_{\mathrm{LPM}}^{\mathrm{q}}$, we get a suppression factor $\eta$ (integral of the Bethe-Heitler simulated spectrum divided by the data) $\eta_{\text {low }}=1.271 \pm 0.138$ and $\eta_{\text {high }}=0.991 \pm 0.121$. Here the latter is clearly consistent with 1 while the former indicates a strong suppression. Furthermore, for the upper $20 \%$ of the spectrum, $\eta_{20}=1.001 \pm 0.087$ which shows no sign of a compensating effect. The numbers for 207 and $149 \mathrm{GeV}$ are similar, showing $\eta$ significantly higher than 1 at low energies compared to $\hbar \omega_{\text {LPM }}^{\mathrm{q}}$ while being slightly higher than, but consistent with, 1 at higher energies. A $\chi^{2}$ analysis for simulations of $287 \mathrm{GeV} e^{-}$in Ir with $E_{\mathrm{LPM}}$ as a free parameter yields $E_{\mathrm{LPM}}=2.2 \pm 0.1 \mathrm{TeV}$ with similar values for 207 and $149 \mathrm{GeV}$, confirming an increase in effective radiation length; see [18].

The possible compensation effect that could counteract the LPM suppression discussed by Bell [17] is thus not observed in the presented data. If his treatment is correct-although doubts have been raised; see, e.g., Ref. [21] — we thus see evidence that "as an essentially quantum effect the increase of penetrating power" must be present.

In conclusion, we have, for the first time, experimentally confirmed the fact that "the penetrability of electrons and positrons increases when $E>E_{0}$ " (where $E_{0}$ is equal to $E_{\mathrm{LPM}}$ in modern notation) [4]. Thus, as the energy of electrons is increased in the neighborhood of $E_{\mathrm{LPM}}$, the effective radiation length increases as well. Good agreement between the experimental data and simulations based on the theoretical expression derived by Migdal [3] has been shown for essentially the complete range of photon energies.

Finally, we have investigated the targets $\mathrm{Ir}, \mathrm{Ta}$, and $\mathrm{Cu}$ for three energies, 287, 207, and $149 \mathrm{GeV}$ in order to examine the detailed behavior, in particular, the depen- dence on energy and target density. These results will appear later [19] together with a more extensive overview of the experimental details.

It is a pleasure to acknowledge the meticulous work of P. B. Christensen on the data-taking system as well as the support from the SL-EA group at CERN, notably N. Doble and P. Pierre. H. D. H. and U. I. U. acknowledge support from the ICE center and Steno, both funded by the Danish Natural Science Research Council.

[1] E. L. Feinberg, Priroda 11, 30 (1994) [English translation available at http://www.phys.au.dk/ ulrik/lpm/ Nature.doc].

[2] K. Kirsebom et al., Nucl. Instrum. Methods Phys. Res., Sect. B 174, 274 (2001).

[3] A. B. Migdal, Sov. Phys. JETP 5, 527 (1957); Phys. Rev. 103, 1811 (1956).

[4] L. Landau and I. Pomeranchuk, Dokl. Akad. Nauk SSSR 92, 535 (1953) [English translation: L. Landau, The Collected Papers of L. D. Landau (Pergamon, New York, 1965), p. 589].

[5] P. L. Anthony et al., Phys. Rev. D 56, 1373 (1997); P. L. Anthony et al., Phys. Rev. Lett. 76, 3550 (1995); P. L. Anthony et al., Phys. Rev. Lett. 75, 1949 (1995).

[6] M. Takeda et al., Phys. Rev. Lett. 81, 1163 (1998).

[7] X. Bertou, P. Billoir, and S. Dagoret-Campagne, Astropart. Phys. 14, 121 (2000).

[8] A. H. Sørensen, Z. Phys. C: Part. Fields 53, 595 (1992).

[9] B. Kämpfer and O. P. Pavlenko, Phys. Lett. B 477, 171 (2000); R. Baier, Yu. L. Dokshitzer, S. Peigne, and D. Schiff, Phys. Lett. B 345, 277 (1995).

[10] V. N. Baier and V. M. Katkov, Phys. Rev. D 57, 3146 (1998); 59, 056003 (1999); 60, 076001 (1999); 62, 036008 (2000).

[11] R. Blankenbecler and S. D. Drell, Phys. Rev. D 53, 6265 (1996).

[12] B. G. Zakharov, Phys. At. Nucl. 62, 1008 (1999).

[13] N. F. Shul'ga and S. P. Fomin, Nucl. Instrum. Methods Phys. Res., Sect. B 145, 73 (1998).

[14] S. Klein, Rev. Mod. Phys. 71, 1501 (1999).

[15] M. L. Ter-Mikaelian, High-Energy Electromagnetic Processes in Condensed Media (Wiley Interscience, New York, 1972).

[16] T. Stanev et al., Phys. Rev. D 25, 1291 (1982).

[17] J.S. Bell, Nucl. Phys. 8, 613 (1958).

[18] N. F. Shul'ga and S. P. Fomin, JETP 86, 32 (1998).

[19] H. D. Hansen et al., Phys. Rev. D (to be published).

[20] R. Brun et al., GEANT: Detector Description and Simulation Tool, CERN program library long writeup W5013.

[21] Y. Tsai, Rev. Mod. Phys. 46, 815 (1974).

[22] S. R. Klein et al., Proceedings of the XVI International Symposium on Lepton and Photon Interactions at High Energies, edited by P. Drell and D. Rubin (American Institute of Physics, New York, 1994). 